\title{
Polyelectrolyte-Based Platforms for the Delivery of Peptides and Proteins
}

Lili Zhao, ${ }^{a}$ Mariusz Skwarczynski, ${ }^{\mathrm{a}, *}$ Istvan Toth ${ }^{\mathrm{a}, \mathrm{b}, \mathrm{c}, *}$

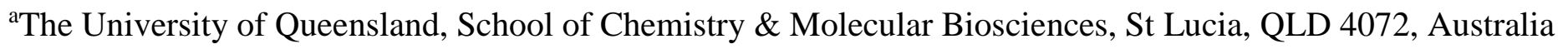

${ }^{\mathrm{b}}$ The University of Queensland, School of Pharmacy, Woolloongabba, QLD 4102, Australia

${ }^{\mathrm{c}}$ The University of Queensland, Institute of Molecular Biosciences, St Lucia, QLD 4072, Australia.

*Corresponding authors:

Professor Istvan Toth,

Dr Mariusz Skwarczynski

The University of Queensland, School of Chemistry and Molecular Biosciences, St Lucia, QLD 4072, Australia. Tel.: +61 733469892 ; Fax: +61 733654273

Email: i.toth@uq.edu.au

m.skwarczynski@uq.edu.au 
Table S1. Peptide and protein-based drugs and vaccines approved by FDA in the recent years

\begin{tabular}{|c|c|c|c|c|}
\hline Generic name & Trade name & Approval date & Company & Therapeutic area \\
\hline Risankizumab-rzaa & Skyrizi & Apr-2019 & AbbVie & plaque psoriasis \\
\hline $\begin{array}{l}\text { Ramucirumab } \\
\text { Immune Globulin Intravenous, Human - slra }\end{array}$ & Cyramza & May-2019 & Eli Lilly & hepatocellular carcinoma with elevated alpha-fetoprotein \\
\hline $10 \%$ Liquid & Asceniv & Apr-2019 & ADMA Biologics & primary Humoral Immunodeficiency Disease \\
\hline Certolizumab pegol & Cimzia & Mar-2019 & $\mathrm{UCB}$ & $\begin{array}{l}\text { non-radiographic axial spondyloarthritis } \\
\text { osteoporosis in postmenopausal women at high risk for }\end{array}$ \\
\hline Romosozumab-aqqg & Evenity & Apr-2019 & Amgen & fracture \\
\hline Pembrolizumab & Keytruda & Apr-2019 & Merk & advanced renal cell carcinoma \\
\hline Trastuzumab and hyaluronidase-oysk & Herceptin Hylecta & Feb-2019 & Halozyme & HER2-overexpressing breast cancer \\
\hline Atezolizumab & Tecentriq & Mar-2019 & Genentech/Roche & triple negative breast cancer \\
\hline Avelumab plus axitinib & Bavencio plus Inlyta & May-2019 & Merck and Pfizer & advanced renal cell carcinoma \\
\hline $\begin{array}{l}\text { Antihemophilic factor (recombinant), } \\
\text { glycopegylated-exei }\end{array}$ & Esperoct & Feb-2019 & Novo Nordisk & hemophilia A \\
\hline Caplacizumab-yhdp & Cablivi & Feb-2019 & Ablynx & acquired thrombotic thrombocytopenic purpura \\
\hline Ibalizumab-uiyk & Trogarzo & Mar-2018 & TaiMed Biologics & human immunodeficiency virus type 1 (HIV-1) infection \\
\hline Tildrakizumab-asmn & Ilumya & Mar-2018 & Sun Pharmaceuticals & plaque psoriasis \\
\hline Erenumab-aooe & Aimovig & May-2018 & Amgen & migraine \\
\hline Coagulation factor $\mathrm{Xa}$ (recombinant) & Andexxa & May-2018 & Portola Pharmaceuticals & hemostasis \\
\hline Burosumab-twza & Crysvita & Apr-2018 & Ultragenyx & X-linked hypophosphatemia (XLH) \\
\hline Avelumab & Bavencio & Mar-2017 & EMD Serono/Pfizer & metastatic Merkel cell carcinoma \\
\hline Dupilumab & Dupixent & Mar-2017 & Regeneron Pharmaceuticals & atopic dermatitis \\
\hline Brodalumab & Siliq & Feb-2017 & Valeant Pharmaceuticals & plaque psoriasis \\
\hline $\begin{array}{l}\text { Tocilizumab } \\
\text { Hepatitis B Vaccine (Recombinant) }\end{array}$ & Actemra & Sep-2017 & Genentech & cytokine release syndrome \\
\hline Adjuvanted & Heplisav-B & Nov-2017 & Dynavax & hepatitis B virus caused infection \\
\hline $\begin{array}{l}\text { Ocrelizumab } \\
\text { C1 Esterase Inhibitor Subcutaneous }\end{array}$ & Ocrevus & Mar-2017 & Genentech & sclerosis \\
\hline [Human] & Haegarda & Jun-2017 & CSL Behring & prevent Hereditary Angioedema (HAE) attacks \\
\hline Guselkumab & Tremfya & Jul-2017 & Janssen Biotech & plaque psoriasis \\
\hline Rabies Immune Globulin (Human) & KedRab & Aug-2017 & Kedrion Biopharma & rabies infection \\
\hline Zoster Vaccine Recombinant, Adjuvanted & Shingrix & Oct-2017 & GlaxoSmithKline & prevention of herpes zoster (shingles) \\
\hline Etelcalcetide & Parsabiv & Feb-2017 & Amgen & secondary hyperparathyroidism \\
\hline Semaglutide & Ozempic & Dec-2017 & Novo Nordisk & type 2 diabetes \\
\hline
\end{tabular}




\begin{tabular}{|c|c|c|c|c|}
\hline Generic name & Trade name & Approval date & Company & Therapeutic area \\
\hline Plecanatide & Trulance & Jan-2017 & Synergy Pharmaceuticals & chronic idiopathic constipation \\
\hline Vestronidase alfa-vjbk & Mepsevii & Nov-2017 & Ultragenyx & Mucopolysaccharidosis VII \\
\hline Inotuzumab ozogamicin & Besponsa & Aug-2017 & Pfizer & acute lymphoblastic leukemia. \\
\hline angiotensin II & Giapreza & Dec-2017 & La Jolla Pharmaceuticals & septic shock \\
\hline Emicizumab-kxwh & Hemlibra & Nov-2017 & Genentech & $\begin{array}{l}\text { prevention or reduction of bleeding episodes in patients with } \\
\text { hemophilia A }\end{array}$ \\
\hline Coagulation Factor IX (Recombinant) & Rebinyn & Jun-2017 & Novo Nordisk & hemophilia B \\
\hline Sarilumab & Kevzara & May-2017 & Sanofi & rheumatoid arthritis \\
\hline Ocrelizumab & Ocrevus & Mar-2017 & Genentech & sclerosis \\
\hline Abaloparatide & Tymlos & Apr-2017 & Radius Health & osteoporosis \\
\hline Avelumab & Bavencio & Mar-2017 & EMD Serono/Pfizer & metastatic Merkel cell carcinoma \\
\hline Durvalumab & Imfinzi & May-2017 & AstraZeneca & $\begin{array}{l}\text { advanced or metastatic urothelial carcinoma and Stage III non- } \\
\text { small cell lung cancer }\end{array}$ \\
\hline Ixekizumab & Taltz & Mar-2016 & Eli Lilly & plaque psoriasis and active psoriatic arthritis \\
\hline Lixisenatide & Adlyxin & Jul-2016 & Sanofi Aventis & type II diabetes \\
\hline Bezlotoxumab & Zinplava & Oct-2016 & Merk & recurrent Clostridium difficile infection \\
\hline Nivolumab & Opdivo & May-2016 & Bristol-Myers Squibb & classical Hodgkin lymphoma \\
\hline Antihemophilic Factor (Recombinant) & Kovaltry & Mar-2016 & Bayer & hemophillia A \\
\hline Coagulation Factor IX (Recombinant) & Idelvion & Mar-2016 & CSL Behring & hemophilia B \\
\hline Antihemophilic Factor (Recombinant) & Afstyla & May-2016 & CSL Behring & hemophillia A \\
\hline Obiltoxaximab & Anthim & Mar-2016 & Elusys Therapeutics & inhalational anthrax \\
\hline Daclizumab & Zinbryta & May-2016 & Biogen & sclerosis \\
\hline Olaratumab & Lartruvo & Oct-2016 & Eli Lilly & soft tissue sarcoma \\
\hline Pembrolizumab & Keytruda & Aug-2016 & Merk & head and neck squamous cell cancer \\
\hline Atezolizumab & Tecentriq & May-2016 & Genentech & urothelial carcinoma and metastatic non-small cell lung cancer \\
\hline Adalimumab & Humira & Jul-2016 & Abbvie & uveitis \\
\hline Reslizumab & Cinqair & Mar-2016 & Teva Pharmaceuticals & severe asthma \\
\hline
\end{tabular}

